\title{
Der Brokdorf-Beschluss des Bundesverfassungsgerichts 1985
}

Eine Veröffentlichung aus dem Arbeitskreis für Rechtswissenschaft und Zeitgeschichte an der Akademie der Wissenschaften und der Literatur Mainz

Anselm Doering-Manteuffel, Bernd Greiner und

Oliver Lepsius

\section{Der Brokdorf- Beschluss}

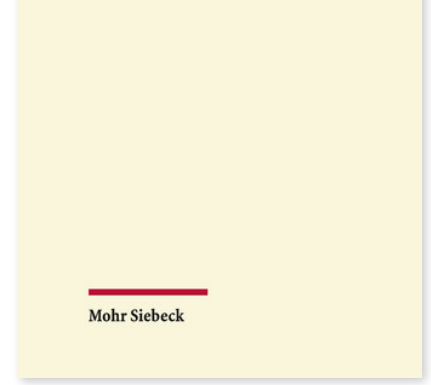

2015. X, 230 Seiten.

ISBN 978-3-16-153762-2

DOI 10.1628/978-3-16-153762-2

eBook PDF $29,00 €$

ISBN 978-3-16-153745-5

Festeinband $29,00 €$
Der Beschluss des Bundesverfassungsgerichts vom 14. Mai 1985 über Verbot oder Zulässigkeit von Demonstrationen gegen den Bau des Kernkraftwerks Brokdorf stellt eine zeitgeschichtliche Quelle ersten Ranges dar. Der Text bündelt Belastungen aus der deutschen Geschichte seit der Zwischenkriegszeit und die hartnäckige Auseinandersetzung um 1980 über die Grenzen der Demonstrationsfreiheit. Bestimmungsmerkmale der freiheitlichen Demokratie und des demokratischen Rechtsstaats wurden neu verhandelt, um dem tiefgreifenden Wandel in der Gesellschaft und dem Anspruch auf Mitbestimmung Rechnung zu tragen. Seit den 1960er Jahren waren nicht nur neue Formen des Bürgerprotests entstanden, hatte sich nicht nur die Wohlstandsgesellschaft entfaltet, sondern es gab auch neue soziale und umweltpolitische Herausforderungen. Sie zogen Massenkundgebungen nach sich, die von den staatlichen Instanzen Polizei, Verwaltung und Gerichtswesen nicht angemessen bewältigt werden konnten. Im Zuge der Studentenbewegung seit Mitte der 1960er Jahre und nach dem Aufbruch der Neuen Sozialen Bewegungen im darauffolgenden Jahrzehnt war es längst an der Zeit, das Versammlungsrecht zu reformieren. Die Legislative hatte sich dieser Aufgabe nicht gestellt, so dass bei den politisch und ökologisch motivierten Massenkundgebungen der 1970er Jahre die neuen Formen des Protests und die alten Verhaltensmuster der Ordnungskräfte unvermittelt aufeinanderprallten. Der Band untersucht aus historischer, juristischer und kulturanthropologischer Perspektive die Bedingungen dieses Wandels und erklärt die Eigenart des verfassungsgerichtlichen Urteils.

Anselm Doering-Manteuffel Geboren 1949; Studium der Geschichte, Germanistik, Kunstgeschichte und Politikwissenschaft; 1980 Promotion; 1986 Habilitation; seit 1991 Direktor des Seminars für Zeitgeschichte der Universität Tübingen; seit 2008 Ordentliches Mitglied der Akademie der Wissenschaften und der Literatur Mainz.

Bernd Greiner Geboren 1952; Studium der Geschichte, Politikwissenschaft und Amerikanistik; 1984 Promotion; seit 1989 im Hamburger Institut für Sozialforschung; 1997 Habilitation; seit 2004 Professor am Fachbereich Geschichtswissenschaft der Universität Hamburg.

Oliver Lepsius ist Professor für Öffentliches Recht und Verfassungstheorie an der Universität Münster.

Jetzt bestellen:

https://mohrsiebeck.com/buch/der-brokdorf-beschluss-des-bundesverfassungsgerichts-1985-9783161537622?no_cache=1 order@mohrsiebeck.com

Telefon: +49 (0)7071-923-17

Telefax: $+49(0) 7071-51104$ 